\title{
Antithrombotic mechanism of polysaccharides in Blackberry (Rubus spp.) seeds
}

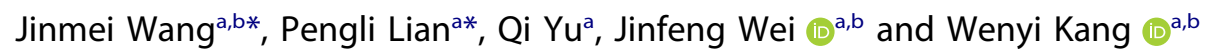

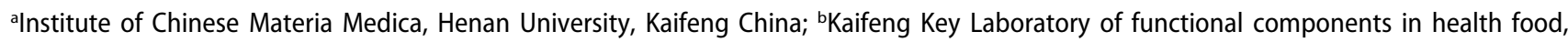 \\ Kaifeng China
}

\begin{abstract}
The blackberry seed was typically removed as a byproduct and waste from blackberry fruits for juices. Developing value-added utilization of berry seeds will significantly expand the market for berry products as well as improve benefit to berry producers. However, the research on blackberry seed is limited. The objective of this paper was to research antithrombotic mechanism of polysaccharides in blackberry seeds. Polysaccharides in blackberry seeds were extracted, purified and identified by high-performance gel permeation chromatography (HPSEC), gas chromatography (GC), fourier transform infrared (FT-IR) spectrometer and nuclear magnetic resonance spectra (NMR). Anticoagulant activities were evaluated in vivo by measuring activated partial thromboplastin time (APTT), thrombin time (TT), prothrombin time (PT), fibrinogen (FIB) and plasma recalcification time (RRT). Four polysaccharides named BSP-1a, BSP-1b, BSP-2 and BSP-3 were isolated from Blackberry (Rubus spp.) seeds. The results indicated that BSP-1b, BSP-2 and BSP-3 exhibited the anticoagulant activity. Therefore, the anti-thrombosis effects of BSP-1b, BSP-2 and BSP-3 were investigated in vivo by 6-Keto-PGF ${ }_{1 a}$, thromboxane $B_{2}\left(T_{X B_{2}}\right)$, endothelial nitric oxide synthase (eNOS), endothelin-1 (ET-1), whole blood viscosity (WBV), plasma viscosity (PV), hematocrit (Hct), erythrocyte sedimentation rate (ESR), APTT, TT, PT and FIB. The results suggested that BSP-1b, BSP-2 and BSP-3 had the inhibition effect on thrombus formation, and the antithrombotic effects were associated with the regulation of vascular endothelium active substance, activating blood flow and anticoagulation effect.
\end{abstract}

\section{ARTICLE HISTORY}

Received 24 May 2017 Accepted 10 September 2017

\section{KEYWORDS}

Blackberry seed;

polysaccharides; structural;

anticoagulant;

antithrombotic

\section{Introduction}

Blackberry (Rubus spp.) is a widely distributed shrub with high commercial value due to its sensory and chemical characteristics,such as sugars, malic acid, amino acids, VC, VE, anthocyanins, superoxide dismutase (SOD) and flavonoids [1]. Therefore, it is usually used as raw material for the production of jams, candy, wine and juice beverages, and so on. The seeds of the blackberry fruits were typically removed during juice processing. In our previous research, 13 compounds were isolated and identified from blackberry seeds, pharmacology research indicated that blackberry seeds had antioxidant [2], cholesterol-lowering [3], liver-protection effects [4], and could rectify the derangement in lipide metabolism [5]. However, there is no research on polysaccharides from blackberry seeds in literature.

Plant polysaccharides display a wide variety of biological activity, including immunostimulatory [6], anticancer [7], anti-diabetic [8] and antithrombotic activities [9]. As already shown for algae polysaccharides, so do plant polysaccharides have anticoagulant activity [10-12], and can be used as a prevention and treatment of cardiovascular disease [13]. The aim of this study was to isolate, characterize and evaluate the anticoagulant and antithrombotic effects of the polysaccharides of blackberry seeds. The results may provide a reference for the further research and development of Blackberry (Rubus spp.).

\section{Materials and methods}

\section{Plant materials}

Seeds of Blackberry (Rubus spp.) were collected in July 2013 from the blackberry planting base of Fengqiu (Henan, China) and were identified by Professor Chang-qin Li in the Department of Pharmacognosy, Henan University. The voucher specimens were deposited at Traditional Chinese Medicine Research Institute of Henan University.

CONTACT Jinfeng Wei weijinfeng20112011@hotmail.com; Wenyi Kang kangweny@hotmail.com @ Institute of Chinese Materia Medica, Henan University, Kaifeng 475004, China

*These authors contributed equally to this work.

(c) 2017 The Author(s). Published by Informa UK Limited, trading as Taylor \& Francis Group.

This is an Open Access article distributed under the terms of the Creative Commons Attribution License (http://creativecommons.org/licenses/by/4.0/), which permits unrestricted use, distribution, and reproduction in any medium, provided the original work is properly cited. 


\section{Animals}

Male rabbit (2.0-2.5 kg), were provided by traditional Chinese medicine research institute of Henan University.

Female and male Sprague-Dawley (SD) rats (200$250 \mathrm{~g})$ were purchased from Laboratory Animal Center, Zhengzhou, Henan, China. They were maintained under a $12 / 12^{\circ} \mathrm{h}$ light/dark cycle, at $25 \pm 2^{\circ}$, with free access of food and water.

\section{Chemicals}

DEAE-cellulose-52 was purchased from Whatman; Sephadex G-100 was purchased from Pharmacia; Kits for activated partial APTT, PT, TT and FIB were from Shanghai Sun Biotech Co., Ltd; Rat 6 Keto-PGF $1 \alpha$ ELSA kit, rat $\mathrm{TXB}_{2}$ ELSA kit, Rat ET-1 ELSA kit and Rat eNOS ELSA kit were from Nanjing senbeijia biotech Co., Ltd.

\section{Extraction and purification of polysaccharide}

The dried Blackberry seeds (200 g) were ground into powder and defatted three times by petroleum ether at room temperature. The organic solvent was volatilized to obtain a dry powder. Then the powder was extracted three times with $70 \%$ ethanol and filtered. Finally, the residue was lyophilized. Subsequently, the dried powder was extracted with 20 volumes of distilled water at $80^{\circ}$ every $3 \mathrm{~h}$ for three times. The aqueous extract was filtered and the supernatant was treated with 95\% ethanol (final concentration $70 \%$ ) at $4^{\circ}$ overnight, and centrifuged at 10,000 $\mathrm{rpm}$ for $10 \mathrm{~min}$. The precipitation was added with Sevage reagent (chloroform/1-butanol, $1: 4 \mathrm{v} / \mathrm{v}$ ) for deproteinization deproteinisation [14]. The crude polysaccharide was obtained through precipitation with 95\% ethanol (final concentration 70\%) and centrifuged. Then the precipitate was redissolved in water and dialyzed for 2 days to remove small molecular weight impurity (molecular weight cut off $3500 \mathrm{Da})$. Finally, the aqueous extract was lyophilized in vacuum to give the crude polysaccharide $(9.7 \mathrm{~g})$.

The $300 \mathrm{mg}$ crude polysaccharides were dissolved in $10 \mathrm{~mL}$ distilled water, and then the water was filtered through $0.45 \mu \mathrm{m}$ microporous membrane. Then the filtered water was fractioned by DEAE-52 column $(2.5 \times 60 \mathrm{~cm})$. The column was eluted with distilled water at $0.8 \mathrm{~mL} \cdot \mathrm{min}^{-1}$ followed by $0.1 \mathrm{M} \mathrm{NaCl}$ and $0.2 \mathrm{M} \mathrm{NaCl}$, respectively. The fractions were collected using an automated step-by-step fraction collector and guided for total carbohydrate using the phenol-sulfuric acid method [15]. Three main fractions were collected, dialyzed, lyophilized and named as BP-1(74 mg), BP-2 (104 mg)and BP-3(95 mg). These polysaccharides were further purified through a column of Sephadex G-100 $(1.5 \times 100 \mathrm{~cm})$ and eluted with water at $0.5 \mathrm{~mL} \cdot \mathrm{min}^{-1}$. The purified fraction was combined, concentrated and lyophilized for further study.

Moreover, four polysaccharides were analysed for concents of total carbohydrates, protein and uronic acid using phenol-sulfuric acid, bradford [16] and m-hydroxydiphenyl [17].

\section{Molecular weight analysis}

The molecular weight of polysaccharides was identified by high-performance size-exclusion chromatography [18]in Beijing centre for physical and chemical analysis.

\section{Monosaccharide composition analysis}

Polysaccharide samples (10 $\mathrm{mg}$ ) were hydrolyzed in ampoules with $2 \mathrm{M}$ trifluoroacetic acid $(2 \mathrm{~mL})$ for $3 \mathrm{~h}$ at $110^{\circ}$, evaporated and added with methanol to remove TFA. Then the hydrolyzates were mixed with $10 \mathrm{mg}$ hydroxylamine hydrochloride and $0.5 \mathrm{~mL}$ pyridine and incubated at $90^{\circ}$ for $30 \mathrm{~min}$. Acetic anhydride $(0.5 \mathrm{~mL})$ was added and incubated at $90^{\circ}$ for $30 \mathrm{~min}$ [19]. The mixtures were cooled to room temperature, and filtered through $0.22 \mu \mathrm{m}$ filters. The resulting alditol acetates were analysed by gas chromatograph (GC), which was performed on a Thermo TRACE1300 instrument (Milan, Italy) fitted with $\operatorname{FID}\left(280^{\circ}\right)$ and equipped with TGWaxms column $(30 \mathrm{~m} \times 0.32 \mathrm{~mm} \times 0.5 \mu \mathrm{m})$. The column temperature was maintained at $100^{\circ}$ for $1 \mathrm{~min}$, and increased to $230^{\circ}$ for $10 \mathrm{~min}$ at a rate of $4^{\circ} \cdot \mathrm{min}^{-1}$ with $\mathrm{N}_{2}$ as the carrier gas. The standard monosaccharides were prepared and subjected to GC analysis separately in the same way.

\section{Spectroscopic analysis}

The infrared spectrum of four polysaccharides were measured on a FT-IR spectrometer (Vertex 70, Bruker, Germany) using $\mathrm{KBr}$ pellets in the infrared region of $4000-500 \mathrm{~cm}^{-1}$. ${ }^{1} \mathrm{H}$ NMR analyses of them were carried out on an Avance Bruker III HD $400 \mathrm{MHz}$ NMR spectrometer.

\section{Anticoagulation assays in vitro}

\section{Anticoagulation time assay}

Anticoagulation activities of APTT, PT, TT and FIB were performed in vitro and the assay was conducted by using rabbit blood collected from rabbit ear veins in plastic 
tubes containing $3.8 \%$ sodium citrate (citrate/blood: $1 / 9$, $\mathrm{v} / \mathrm{v})$. Then, the blood was centrifuged at $3000 \mathrm{rpm}$ for $15 \mathrm{~min}$ at $5^{\circ} \mathrm{C}$ to obtain the serums. For APTT assay, $25 \mu \mathrm{L}$ of sample solution tested was mixed with $50 \mu \mathrm{L}$ of citrated normal rabbit serum, and then APTT assay reagent was added. Following, the mixture was incubated at $37^{\circ} \mathrm{C}$ for $5 \mathrm{~min}$. Then $25 \mathrm{mM} \mathrm{CaCl}_{2}$ solution $(100 \mu \mathrm{L})$ was added into the incubated mixture to initiate the reaction. Finally, the clotting time was recorded. For PT assay, samples $(25 \mu \mathrm{L})$ were mixed with serum $(25 \mu \mathrm{L})$ and incubated at $37^{\circ} \mathrm{C}$ for $3 \mathrm{~min}$. While PT assay reagent $(50 \mu \mathrm{L})$, which had been hatched for $10 \mathrm{~min}$ at $37^{\circ} \mathrm{C}$, was then added and clotting time was recorded. TT and FIB assays were performed according to the manufacturer's specifications [20]. For all clotting assays, blank solvent was used as the blank control group, and breviscapine and Vitamin $\mathrm{K}_{1}$ were used as the positive control group, and the time for clot formation was recorded by a SemiAutomated Coagulation Analyser (Han Fang, Jinan, China).

\section{PRT assays in vitro}

Blood samples were collected from rabbit ear veins in the plastic tubes containing $3.8 \%$ sodium citrate (citrate/blood: 1/9, v/v). Then, the blood was centrifuged at $1000 \mathrm{rpm}$ for $15 \mathrm{~min}$ to obtain the plasma. Tested samples $(100 \mu \mathrm{L})$ were mixed with $100 \mu \mathrm{L}$ of citrated normal rabbit plasma, and then RPT assay reagent was added. All experimental groups were adding $0.1 \mathrm{~mL}$ of $\mathrm{CaCl}_{2}$ solution $(0.925 \mathrm{mg} / \mathrm{mL})$ after training $1 \mathrm{~min}$ at $37^{\circ}$. The control group, coagulant control and anti-coagulant control were repeated three times, the sample group was repeated six times [21].

\section{Anti-thrombosis activity assays in vivo}

\section{Experimental model}

The SD rats were randomly divided into seven groups with six animals in each. The groups included the blank control group, model group, positive group and three-treated group. The rats of the blank control group and model group were given the corresponding blank solvent (distilled water) by gavage. The positive control group was given aspirin $(100 \mathrm{mg} / \mathrm{kg})$. The three treated groups were given BSP-1b $(120 \mathrm{mg} / \mathrm{kg})$, BSP-2 $(120 \mathrm{mg} / \mathrm{kg})$ and BSP-3 $(120 \mathrm{mg} / \mathrm{kg})$, respectively. All of the groups were given by gavage twice a day for 7 days.

After the fifth administration, blood stasis model was established, except for the blank control rats. The model rats were injected with adrenaline hydrochloride $(0.8 \mathrm{mg} / \mathrm{kg})$. After $2 \mathrm{~h}$ of the first administration of epinephrine hydrochloride subcutaneously, the rats were placed in ice water (from 0 to $2^{\circ}$ ) for $5 \mathrm{~min}$, and then injected with adrenaline hydrochloride $(0.8 \mathrm{mg} / \mathrm{kg})$ again $2 \mathrm{~h}$ after the icebath. Subsequently, rats were fasted for $12 \mathrm{~h}$ with free access to water and administration continued.

The rats were anesthetized with $10 \%$ chloral hydrate $(300 \mathrm{mg} / \mathrm{kg}) 30 \mathrm{~min}$ after the last administration. Then the blood samples were collected from abdominal aorta to determine the corresponding parameters.

\section{Plasma coagulation parameters in vivo}

Blood samples were collected from abdominal aorta into the plastic tubes containing $3.8 \%$ sodium citrate (citrate/blood: 1/9, v/v). Then, the blood was centrifuged at $1000 \mathrm{rpm}$ for $15 \mathrm{~min}$ to obtain the plasma. The determination method for APTT, PT, TT and FIB accorded with anticoagulation assays in vitro.

\section{Determination of TXB2, 6-keto-PGF1a, eNOS and ET-1}

The preparation method of the plasma was reference to plasma coagulation parameters in vivo. The plasmas of $\mathrm{TXB}_{2}$, 6-keto-PGF $\mathrm{Pa}_{1 \alpha}$, eNOS and ET-1 were performed by enzyme-linked immunoassay according to the manufacturer's recommended procedures.

\section{Hemorheology parameters}

The WBV and PV of the samples were measured by a Auto-Viscometer. For WBV, the blood samples mixed with heparin sodium were placed into the sample cell of the Auto-Viscometer. Then, WBV was measured with shear rates of $200 / \mathrm{s}, 20 / \mathrm{s}$ and $3 / \mathrm{s}$ at $37^{\circ} \mathrm{C}$. For $\mathrm{PV}$, the blood was centrifuged at $1000 \mathrm{rpm}$ for $15 \mathrm{~min}$ to obtain the plasma, and then PV was measured by the viscometer. Hct was determined by the Wintrobe method and ESR was determined by Westergren method.

\section{Statistical analysis}

All experimental results were expressed as mean \pm standard deviation (SD). Statistical analysis was performed with the SPSS19.0 software. Comparison between any two groups was evaluated using one-way analysis of variance (ANOVA). 


\section{Results and discussion}

\section{Extraction and purification of polysaccharide}

Crude polysaccharides were successfully isolated by a series of experimental procedures such as water extraction, deproteination, dialysis, ethanol precipitation and lyophilization. The crude polysaccharides were then separated by using DEAE-cellulose- 52 column and further purified by Sephadex G-100. As a result, four purified fractions were obtained, named as BSP-1a (34 mg), BSP-1b(21 mg), BSP-2(50 mg) and BSP-3 (46 mg). BSP-1a contained $96.31 \%$ of total carbohydrate and $1.58 \%$ of protein. BSP-1b contained $94.29 \%$ of total carbohydrate and $2.71 \%$ of protein. BSP-2 contained $93.76 \%$ of total carbohydrate, $4.06 \%$ of protein and $8.91 \%$ of uronic acid. BSP-3 contained $92.35 \%$ of total carbohydrate, $2.12 \%$ of protein and $7.22 \%$ of uronic acid.

\section{Molecular weight analysis}

Molecular weight of polysaccharide was a statistical average, which was representative of similar polymer chain length distributed on average. The average molecular weight $(M w)$ of the four polysaccharides was $16,868, \quad 178,500, \quad 33,600$ and $224,300 \mathrm{~g} / \mathrm{mol}$, respectively.

\section{GC analysis}

The monosaccharide composition of BSP-1a was galactose, fructose, rhamnose, arabinose and xylose in a molar ratio of $1.42: 1.48: 1.35: 19.59: 7.83$. BSP-1b was composed of galactose, fructose, rhamnose, arabinose and xylose with a molar ratio of 19.13:2.27:3.30:5.48:1.78. The monosaccharide composition of BSP-2 was determined to be galactose, fructose, rhamnose, arabinose and xylose in a ratio of 2.55:1.95:7.40:17.98:8.71. BSP-3 was composed of galactose, fructose, rhamnose and arabinose in a ratio of 0.58:0.91:3.41:2.63.

\section{FT-IR}

The FT-IR spectroscopy of BSP-1a, BSP-1b, BSP-2 and BSP-3 were scanned between 4000 and $500 \mathrm{~cm}^{-1}$ and the results showed the four polysaccharides were similar to each other (Figure 1). The absorption band was found in all samples between $3351 \mathrm{~cm}^{-1}$ and $3417 \mathrm{~cm}^{-1}$, indicating the presence of hydroxyl group. The appearance of the peaks within the range of $2800-2950 \mathrm{~cm}^{-1}$ was due to the presence of the $\mathrm{C}-\mathrm{H}$ stretching vibration. The signals at around 1597-1654 $\mathrm{cm}^{-1}$ and 1409-1420 were showing the presence of carboxyl groups. Absorption at 1010-1100 the $\mathrm{C}-\mathrm{O}$ and $\mathrm{C}-\mathrm{C}$ stretching vibrations of pyranose ring. Moreover, the characteristic absorption between $820 \mathrm{~cm}^{-1}$ and $890 \mathrm{~cm}^{-1}$ indicated that $\alpha$-glycosidic linkages and $\beta$-glycosidic linkages in the polysaccharides.

\section{${ }^{1} \mathrm{H}$ NMR spectral analysis}

As shown in Figure 2, the anomeric region of the ${ }^{1} \mathrm{H}$ NMR spectrum showed mostly of signals at 4.0-5.5 ppm for the four polysaccharides. The chemical shifts from $\delta 5.0$ to $5.5 \mathrm{ppm}$ were attributed to a-glycosidic linkage, and the signals between $\delta 4.0$ and $5.0 \mathrm{ppm}$

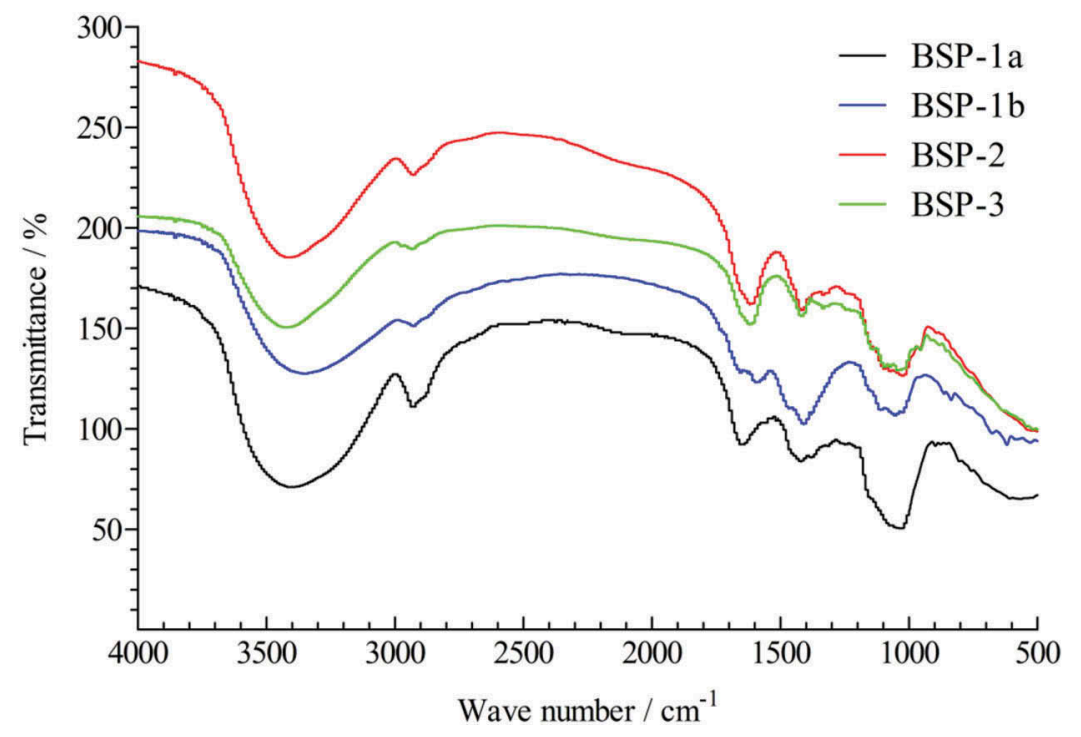

Figure 1. Infrared spectra of BSP-1a, BSP-1b, BSP-2 and BSP-3. 

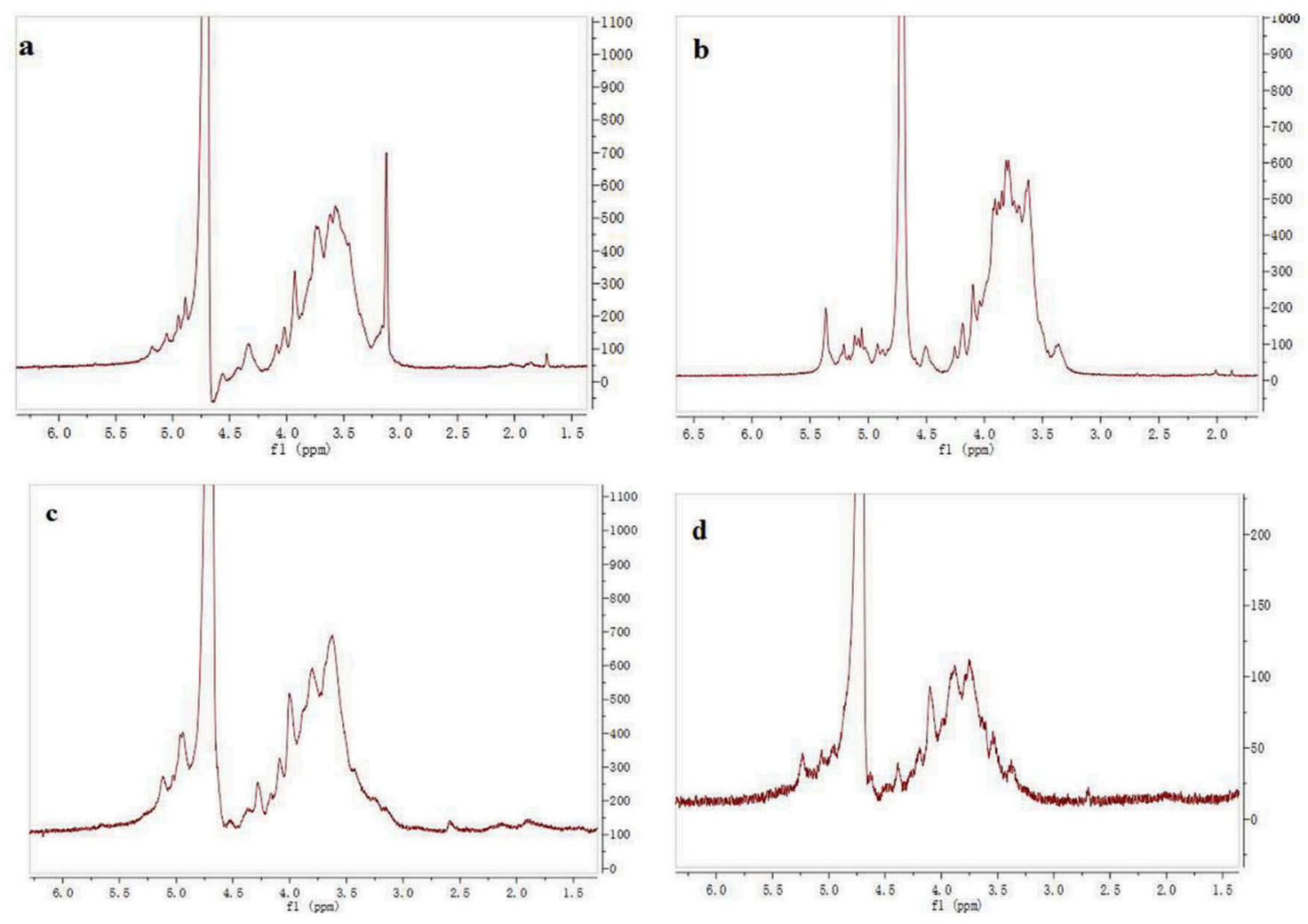

Figure 2. ${ }^{1} \mathrm{H}$ NMR spectrum of BSP-1a (a), BSP-1b (b), BSP-2 (c) and BSP-3 (d).

were assigned to $\beta$-glycosidic linkage. These findings were in accordance with FT-IR spectrum.

\section{Anticoagulation assays in vitro}

The anticoagulant assays of BSP-1a, BSP-1b, BSP-2 and BSP-3 were tested by PT, APTT, TT, FIB and PRT in vitro. $\mathrm{PT}$ is used to evaluate the coagulation factors $\mathrm{V}$, VII and $\mathrm{X}$ in the overall efficiency of extrinsic clotting pathway. On the other hand, APTT is a test of the coagulation factors VIII, IX, XI, XII and Von Willebrand's factor [22] in the intrinsic clotting activity. TT is mainly a measure of transformation of fibrinogen to fibrin degree. FIB is employed to reflect the content of fibrinogen. The PRT is an important indicator for measuring the activity of the endogenous coagulation process [23]

In Table 1, compared with the blank group, BSP-1a could significantly shorten APTT, PT and TT $(P<0.001)$ and could significantly increase the content of FIB $(P<0.001)$, which indicated that BSP-1a had coagulant activities and exerted the coagulant activities through intrinsic pathway, extrinsic pathway and increased the content of FIB. Compared with the blank group, BSP-1b could significantly prolong PRT, APTT, TT and PT $(0.01<P<0.05$, or $P<0.001$, respectively), so the anticoagulant activities of BSP-1b was associated with the intrinsic pathway and extrinsic pathway. Compared with the blank group, BSP-2 could significantly prolong PRT, APTT and TT $(P<0.001$, respectively) and could significantly decrease the

Table 1. Anticoagulation activity of BSP-1a, BSP-1b, BSP-2 and BSP-3.

\begin{tabular}{|c|c|c|c|c|c|}
\hline Group & PRT(s) & APTT(S) & PT(S) & $\Pi \mathrm{T}(\mathrm{s})$ & $\mathrm{FIB}(\mathrm{g} / \mathrm{L})$ \\
\hline blank & $219.7 \pm 3.2$ & $20.50 \pm 0.08$ & $9.93 \pm 0.10$ & $32.78 \pm 0.10$ & $3.38 \pm 0.02$ \\
\hline breviscapine & $274.5 \pm 5.2^{\# \# \#}$ & $32.73 \pm 0.13^{\# \# \#}$ & $10.95 \pm 0.06^{\# \# \#}$ & $39.55 \pm 0.13^{\# \# \#}$ & $5.29 \pm 0.17^{\# \# \#}$ \\
\hline vitamin k1 & $164.1 \pm 2.0^{\# \# \#}$ & $13.53 \pm 0.13^{\# \# \#}$ & $7.55 \pm 0.06^{\# \# \#}$ & $19.67 \pm 0.17^{\# \# \#}$ & - \\
\hline BPS-1a & $237.7 \pm 3.7^{\# \# \# \Delta \Delta \Delta}$ & $15.68 \pm 0.13^{\# \# \# \Delta \Delta \Delta}$ & $8.63 \pm 0.15^{\# \# \# \Delta \Delta \Delta}$ & $31.25 \pm 0.06^{\# \# \# \Delta \Delta \Delta}$ & $3.83 \pm 0.12^{\# \# \# * * *}$ \\
\hline BPS-1b & $342.0 \pm 4.9^{\# \# \# * * *}$ & $45.70 \pm 0.08^{\# \# \#_{* * * *}}$ & $10.45 \pm 0.42^{\# \# * *}$ & $53.20 \pm 1.40^{\# \# \# \text { *** }}$ & $3.41 \pm 0.09^{\# * * *}$ \\
\hline BPS-2 & $318.8 \pm 3.8^{\# \# \# \text { **** }}$ & $24.15 \pm 0.06^{\# \# \# * * * *}$ & $9.90 \pm 0.14^{\#}$ & $39.65 \pm 1.43^{\# \# \# *}$ & $3.12 \pm 0.14^{\# \# \# * * *}$ \\
\hline BPS-3 & $352.8 \pm 8.8^{\# \# \# \text { **** }}$ & 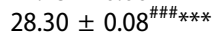 & $10.83 \pm 0.15^{\# \# \# *}$ & $61.50 \pm 3.05^{\# \# \# * * *}$ & $2.65 \pm 0.10^{\# \# \# * * * *}$ \\
\hline
\end{tabular}

Compared with Blank $\left(0.01<{ }^{*} P<0.05,0.001<{ }^{* *} P<0.01,{ }^{* * *} P<0.001\right)$;

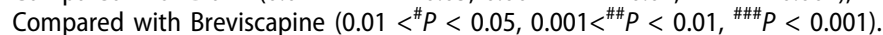

Compared with Vitamin $\mathrm{k} 1\left(0.01<{ }^{\Delta} P<0.05,0.001<{ }^{\Delta \Delta} P<0.01,{ }^{\Delta \Delta \Delta} P<0.001\right)$. 
content of FIB $(P<0.001)$, but PT had no difference with the blank group $(P>0.05)$, and thus suggested that BSP-2 had anticoagulant activities and exerted the anticoagulant activities through intrinsic pathway and increased the content of FIB. So the anticoagulant activities of BSP-2 were associated with the intrinsic pathway and extrinsic pathway. Compared with the blank group, BSP-3 could significantly prolong PRT, APTT, TT and PT $(0.01<P<0.05$, or, $P<0.001$, respectively), and could significantly decrease the content of FIB $(P<0.001)$, which indicated that BSP-3 had anticoagulant activities and exerted the anticoagulant activities through intrinsic pathway, extrinsic pathway and decreased the content of FIB.

\section{Anti-thrombosis activity assays in vivo}

$\mathrm{TXB}_{2}$ is a degradation product of thromboxane $\mathrm{A}_{2}$ $\left(\mathrm{TXA}_{2}\right)$ and 6-keto-PGF ${ }_{1 \alpha}$ is a metabolite of epoprostenol $\left(\mathrm{PGI}_{2}\right) . \mathrm{TXA}_{2}$ and $\mathrm{PGI}_{2}$ are extremely unstable in the body. Thus, to detect the contents of $\mathrm{TXB}_{2}$ and 6keto-PGF ${ }_{1 \alpha}$ can reflect $\mathrm{TXA}_{2}$ and $\mathrm{PGI}_{2}$ levels. Research evidence has indicated that $\mathrm{TXA}_{2}$ is a platelet activating agent, which can promote the formation of thrombus. $\mathrm{PGI}_{2}$ is a platelet function inhibitor, which has a protective effect on the vasospasm caused by platelet aggregation. The balance between biosynthesis of $\mathrm{PGI}_{2}$ and that of $\mathrm{TXA}_{2}$ is important in the prevention of thrombosis [24-27].

As shown in Figure 3, compared with the model group, BSP-1b, BSP-2 and BSP-3 could significantly increase the content of 6-Keto-PGF ${ }_{1 \alpha}$ and reduce the content of $\mathrm{TXB}_{2}(P<0.001)$, indicating that the antithrombotic mechanism of BSP-1b, BSP-2 and BSP-3 was associated with the balance of $\mathrm{TXB}_{2}$ and 6-keto-PGF ${ }_{1 \alpha}$.

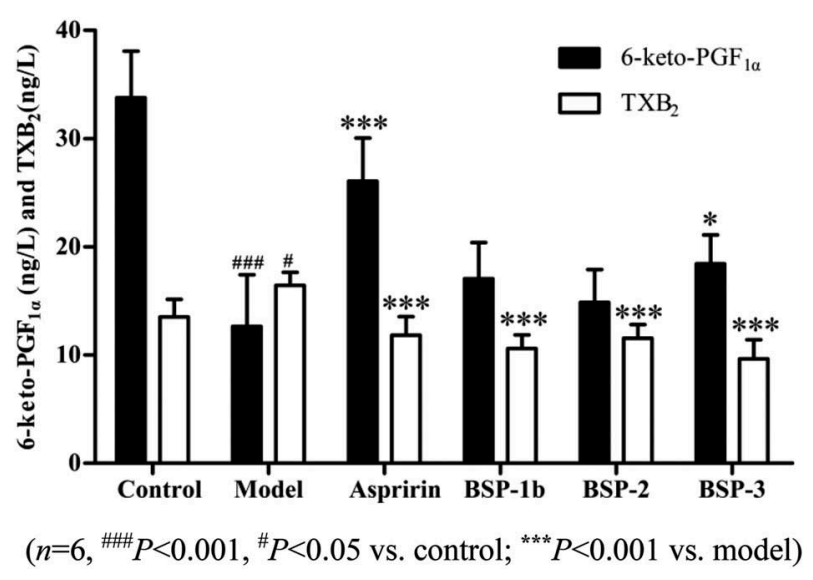

Figure 3. Effect on 6-keto-PGE $1 a$ and TXB2 in rats. ( $n=6$, ${ }^{\# \#} P<0.001,{ }^{\#} P<0.05$ vs. control; ${ }^{* * *} P<0.001$ vs. model).
ET-1, which is widely found in vascular endothelium, plays an important role in cardiovascular function. Vascular contraction, myocardial ischemia, metabolic disorder and cell proliferation induced by ET-1 are the common pathogenic factors of vascular injury. The eNOS can be activated to generate endogenous NO, which can inhibit the formation of thrombosis through regulating the tension of the vascular smooth, inhibiting platelet aggregation and vascular smooth muscle cell proliferation.

In Figures 4 and 5, compared with the model group, BSP-1b, BSP-2 and BSP-3 could significantly reduce the content of ET-1 and could significantly increase the content of eNOS $(P<0.001)$. The result indicated that BSP-1b, BSP-2 and BSP-3 could inhibit the formation of thrombosis by regulating the content of ET-1 and eNOS [28].

WBV reflects the intrinsic resistance of hemokinesis in blood vessels and it varies according to the different shear rate. The abnormality of WBV can increase the

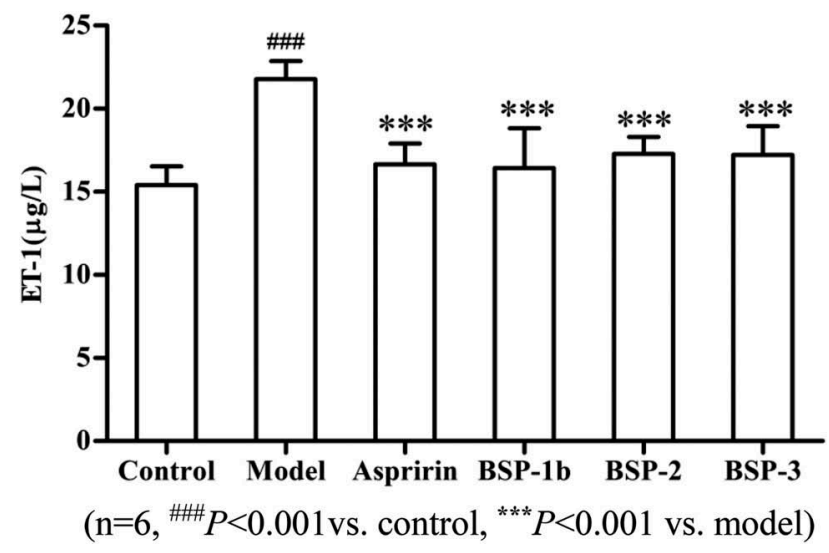

Figure 4. Effect on ET-1 in rats.

( $\mathrm{n}=6,{ }^{\# \# \#} P<0.001$ vs. control, ${ }^{* * *} P<0.001$ vs. model).

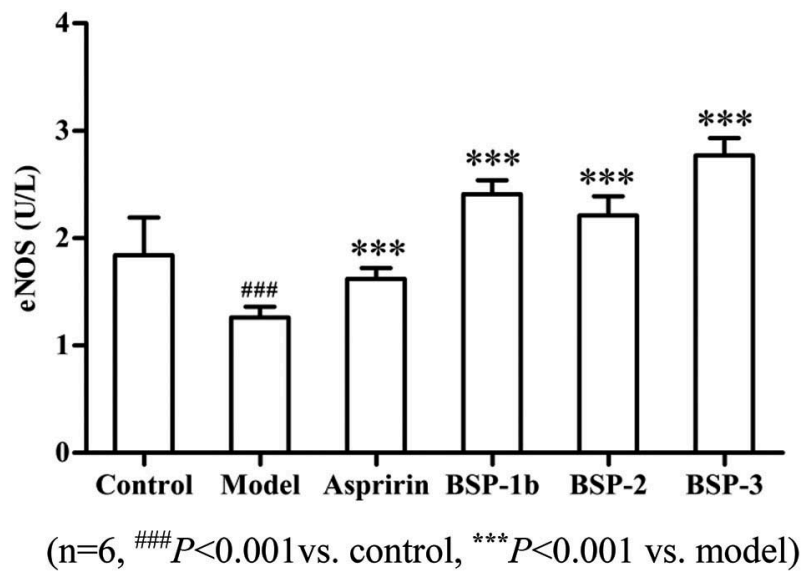

Figure 5. Effect on eNOS in rats. ( $\mathrm{n}=6,{ }^{\# \# \#} P<0.001$ vs. control, ${ }^{* * *} P<0.001$ vs. model). 
Table 2. Effect on WBV and Hct.

\begin{tabular}{|c|c|c|c|c|c|}
\hline \multirow[b]{2}{*}{ Group } & \multirow[b]{2}{*}{ Dose $(\mathrm{mg} / \mathrm{kg})$} & \multicolumn{3}{|c|}{$\mathrm{WBV}(\mathrm{mPa} \cdot \mathrm{s})$} & \multirow[b]{2}{*}{$\mathrm{Hct}(\%)$} \\
\hline & & $200 / \mathrm{s}$ & $20 / \mathrm{s}$ & $3 / \mathrm{s}$ & \\
\hline Blank & - & $3.54 \pm 0.31$ & $4.60 \pm 0.29$ & $9.23 \pm 0.13$ & $37.85 \pm 0.93$ \\
\hline Model & - & $4.28 \pm 0.23^{\# \# \#}$ & $5.94 \pm 0.35^{\# \# \#}$ & $11.11 \pm 0.35^{\# \# \#}$ & $41.96 \pm 0.91^{\# \# \#}$ \\
\hline Aspirin & 100 & $3.83 \pm 0.37^{\Delta}$ & $4.78 \pm 0.46^{\Delta \Delta \Delta}$ & $9.53 \pm 0.19^{\Delta \Delta \Delta}$ & $39.62 \pm 0.78^{\Delta \Delta}$ \\
\hline BSP-1b & 120 & $3.80 \pm 0.32^{\Delta}$ & $5.12 \pm 0.14^{\Delta \Delta \Delta}$ & $9.94 \pm 0.44^{\Delta \Delta \Delta}$ & $39.58 \pm 1.36^{\Delta \Delta}$ \\
\hline BSP-2 & 120 & $3.89 \pm 0.20^{\Delta}$ & $5.08 \pm 0.15^{\Delta \Delta \Delta}$ & $10.34 \pm 0.60^{\Delta^{*}}$ & $39.42 \pm 1.56^{\Delta \Delta}$ \\
\hline BSP-3 & 120 & $3.91 \pm 0.29^{\Delta}$ & $5.30 \pm 0.24^{\Delta \Delta}$ & $10.24 \pm 0.79^{\Delta^{* *}}$ & $40.12 \pm 0.62^{\Delta}$ \\
\hline
\end{tabular}

Data represent mean \pm SD. $n=6$;

Compared with blank (\#\#\# $P<0.001$ )

Compared with model $\left({ }^{\Delta \Delta} P<0.001,0.01<{ }^{\Delta \Delta} P<0.01,{ }^{\Delta} P\right.$ 0.05)

Compared with aspirin $\left(0.001<* * P<0.01,{ }^{*} P<0.05\right)$

resistance of blood fluidity and slow down the blood flow velocity, thus leading to the cardiovascular risk. Hct is the most important factor for WBV [29-32]. In Table 2, compared with model group, BSP-1b, BSP-2 and BSP-3 could significantly decrease WBV at all shear rates $(0.001<p<0.05, p<0.001)$, as well as BSP-1b, BSP-2 and BSP-3 could significantly decrease Hct $(p<0.001)$. So BSP-1b, BSP-2 and BSP-3 could reduce the WBV by decreasing Hct. Moreover, PV and ESR play an important role in WBV. The results of ESR and PV are shown in Table 3. Compared with the model group, BSP-1b, BSP-2 and BSP-3 could significantly decrease ESR and PV $(\mathrm{P}<0.001$, or, $0.001<\mathrm{P}<0.01$, or, $\mathrm{P}<0.05)$, suggested that the amelioration effect of administered groups on WBV might be the decrease of ESR and PV.

In Table 4, compared with the model group, BSP-2 and BSP-3 could significantly prolong APTT, PT and

Table 3. Effect on ESR and PV.

\begin{tabular}{lccc}
\hline Group & Dose $(\mathrm{mg} / \mathrm{kg})$ & ESR $(\mathrm{mm} / \mathrm{h})$ & PV $(\mathrm{mPa} \cdot \mathrm{s})$ \\
\hline Blank & - & $3 \pm 0.82$ & $1.36 \pm 0.06$ \\
Model & - & $11.5 \pm 1.29^{\# \# \#}$ & $1.53 \pm 0.09^{\# \#}$ \\
aspirin & 100 & $7 \pm 0.82^{\Delta \Delta \Delta}$ & $1.39 \pm 0.04^{\Delta \Delta}$ \\
BSP-1b & 120 & $4.25 \pm 0.95^{\Delta \Delta \Delta^{* *}}$ & $1.38 \pm 0.07^{\Delta \Delta}$ \\
BSP-2 & 120 & $7.25 \pm 1.7^{\Delta \Delta \Delta}$ & $1.36 \pm 0.09^{\Delta \Delta}$ \\
BSP-3 & 120 & $8.5 \pm 1.3^{\Delta \Delta \Delta}$ & $1.43 \pm 0.05^{\Delta}$ \\
\hline
\end{tabular}

Data represent mean \pm SD. $\mathrm{n}=6$;

Compared with blank $\left.{ }^{\# \# \# P} P<0.001,0.001{ }^{\# \#} P<0.01\right)$

Compared with model $\left({ }^{\Delta \Delta \Delta} P<0.001,0.01<{ }^{\Delta \Delta} p<0.01,{ }^{\Delta} P<0.05\right)$

Compared with aspirin $(0.001<* * P<0.01)$
TT, and could significantly reduce the content of FIB $(\mathrm{P}<0.001)$, suggesting BSP-2 and BSP-3 had the antithrombotic, and the activities were associated with the intrinsic pathway and extrinsic pathway, and decreased the content of FIB. BSP-1b could significantly prolong APTT, PT and TT $(\mathrm{P}<0.001)$, suggesting BSP-1b could inhibit the formation of antithrombotic and the activities were associated with the intrinsic pathway and extrinsic pathway.

\section{Conlusion}

In our study, the crude polysaccharides were obtained from the Blackberry seeds. Sevage reagent and dialysis bag were used to remove proteins and small molecular weight impurity, respectively. Then, BSP-1a, BSP-1b, BSP-2 and BSP-3 were obtained by anion exchange chromatography and sephadex column chromatography. The results of anticoagulant activity of in vitro indicated that BSP-1b, BSP-2 and BSP-3exhibited the anticoagulant activity. Therefore, the anti-thrombosis activity assays of BSP-1b, BSP-2 and BSP-3 were taken for further study. The results suggested that BSP-1b, BSP-2 and BSP-3 had the inhibition effect on thrombus formation in blood stasis model rats, and the antithrombotic activities were associated with the regulation of vascular endothelium active substance, activating blood flow and anticoagulation activity. These findings provided

Table 4. Coagulation parameters of BSP-1a, BSP-1b, BSP-2 and BSP-3.

\begin{tabular}{|c|c|c|c|c|c|}
\hline \multirow[b]{2}{*}{ Group } & \multirow[b]{2}{*}{ Dose $(\mathrm{mg} / \mathrm{kg})$} & \multicolumn{4}{|c|}{ Plasma coagulation parameters } \\
\hline & & APTT(s) & PT(s) & $\mathrm{TT}(\mathrm{s})$ & $\mathrm{FIB}(\mathrm{g} / \mathrm{L})$ \\
\hline blank & - & $44.45 \pm 1.87$ & $23.4 \pm 0.53$ & $36.05 \pm 1.37$ & $2.3 \pm 0.12$ \\
\hline model & - & $23.88 \pm 1.21^{\# \# \#}$ & $18.62 \pm 0.55^{\# \# \#}$ & $18.58 \pm 0.62^{\# \# \#}$ & $3.9 \pm 0.22^{\# \# \#}$ \\
\hline aspirin & 100 & $34.47 \pm 0.63^{\Delta \Delta \Delta}$ & $20.5 \pm 0.52^{\Delta \Delta \Delta}$ & $32.8 \pm 1.11^{\Delta \Delta \Delta}$ & $3.44 \pm 0.13^{\Delta \Delta \Delta}$ \\
\hline BSP-1b & 120 & $39.85 \pm 1.89^{\Delta \Delta \Delta^{* * *}}$ & $24.53 \pm 0.39^{\Delta \Delta \Delta^{* * *}}$ & $28.25 \pm 0.99^{\Delta \Delta \Delta}$ & $3.72 \pm 0.14$ \\
\hline BSP-2 & 120 & $37.19 \pm 1.21^{\Delta \Delta \Delta^{*}}$ & $26.48 \pm 0.92^{\Delta \Delta \Delta^{* * *}}$ & $29.35 \pm 1.06^{\Delta \Delta \Delta}$ & $3.22 \pm 0.23^{\Delta \Delta \Delta}$ \\
\hline BSP-3 & 120 & $38.93 \pm 0.83^{\Delta \Delta \Delta^{* * *}}$ & $27.38 \pm 0.79^{\Delta \Delta \Delta^{* * *}}$ & $29.4 \pm 0.48^{\Delta \Delta \Delta}$ & $2.83 \pm 0.11^{\Delta \Delta \Delta^{* * *}}$ \\
\hline
\end{tabular}

Data represent mean \pm SD. $\mathrm{n}=6$;

Compare with Blank $\left({ }^{\# \#} P<0.001,0.001<{ }^{\#} P<0.05\right)$

Compare with model $\left({ }^{\Delta \Delta \Delta} P<0.001\right)$

Compare with aspirin $\left.{ }^{* * *} P<0.001,{ }^{*} P<0.05\right)$ 
a pharmacological basis for application in clinical of BSP-1b, BSP-2 and BSP-3 on blood stasis syndrome. In addition, these results could be used to create a new industrial field for the production of fish farm supplements, as well as other nutraceuticals [33-36].

\section{Disclosure statement}

No potential conflict of interest was reported by the authors.

\section{Funding}

This work was supported by Henan Province University Science and Technology Innovation Team [16IRTSTHN019], National cooperation project of Henan province [2015GH12], Natural Science Foundaion of Henan Province [162300410038], Science and Technology Innovation Team of Kaifeng City [2016-124], Kaifeng City Science and Technology Innovation Talent [1509010].

\section{ORCID}

Jinfeng Wei (D) http://orcid.org/0000-0001-7608-1036

Wenyi Kang (D) http://orcid.org/0000-0002-1822-6249

\section{References}

[1] Lydia K, Luke RH, Latha D. The blackberry fruit: a review on its composition and chemistry, metabolism and bioavailability, and health benefits. J Agric Food Chem. 2012;60:5716-5727.

[2] Yin $\mathrm{ZH}$, Wang JJ, Gu HP, et al. Antioxidan activity of the fruits of blackberry (Shawnee) in vitro. Nat Prod Res Dev. 2013;25(4):530-532.

[3] Yin ZH, Zhang W, Feng FJ, et al. Study on effective extracts of Rubus spp seed for reducing blood lipid. Chin J Exp Tradit Med Form. 2014;20(24):194-198.

[4] Yin ZH, Zhang W, Feng FJ, et al. Effective extracts of blackberry seed for protection of acute liver injury. Food Sci Technol. 2014;12:94-97.

[5] Zhang W, Yin ZH, Kang WY. Effect of blackberry seed on Alloxan induced diabetes in mice. Sci Technol Food Ind. 2014;19:351-354.

[6] Ji GQ, Chen RQ, Zheng JX. Macrophage activation by polysaccharides from Atractylodes macrocephala Koidz through the nuclear factor- $\mathrm{KB}$ pathway. Pharmaceutical Biolo. 2015;53(4):512-517.

[7] Li C, Cai JP, Geng JS, et al. Purification, characterization and anticancer activity of a polysaccharide from Panax ginseng. Int J Bio Macromol. 2012;51(5):968-973.

[8] Chen X, Jin J, Tang J, et al. Extraction, purification, characterization and hypoglycemic activity of polysaccharide isolated from the root of Ophiopogon japonicas. Carbohydr Polym. 2011;83(2):749-754.

[9] Isak M, Ma H, Zhou WT, et al. Anti-therombotic effect of polysaccharide of Cydonia oblonga Mill and its mechamism. Chin Pharmacological Bull. 2015;31(2):295-296.
[10] Pan XJ, Yang XF, Chen LP, et al. Anticoagulant and fibrinolytic activities of polysaccharides from Curcuma kwangsiensis. Herald Med. 2013;32(2):163-165.

[11] Faggio C, Pagano M, Dottore A, et al. Evaluation of anticoagulant activity of two algal polysaccharides. Nat Prod Res. 2015;30(17):1934-1937.

[12] Faggio C, Morabito M, Minicante SA, et al. Potential use of polysaccharides from the brown alga Undaria pinnatifida as anticoagulants. Braz Arch Biol Technol. 2015;58(5):798-804.

[13] Yoon SJ, Pereira MS, Pavao MSG, et al. The medicinal Plant Porana volubilis contains polysaccharides with anticoagulant activity mediated heparin cofactor II. Thranb Res. 2002;106(1):59-62.

[14] Wang S, Huang SY. Deprotein of plant polysaccharide extract. Food Sci Technol. 2012;37(9):188-191.

[15] Dubois M, Gilles K, Hamilton JK, et al. A colorimetric method for the determination of sugars. Nature. 1951;168:167.

[16] Bradford MM. A rapid and sensitive method for the quantization of microgram quantities of protein utilizing the principle of protein-dye binding. Anal Biochem. 1976;72:248-254.

[17] Blumenkrantz N, Asboe-Hansen G. New method for quantitative determination of uronic acids. Anal Biochem. 1973;54:484-489.

[18] National Pharmacopoeia Committee. Pharmacopoeia of People's Republic of China. Beijing: China Medical Sicence Press; 2010.

[19] Zhang WJ. Biochemical research technology of glycoconjugate. Zhejiang: Zhengjiang University Press; 2006.

[20] $\mathrm{Ku} \mathrm{SK}$, Kim TH, Bae JS. Anticoagulant activities of persicarin and isorhamnetin. Vascul Pharmacol. 2013;58(4):272-279.

[21] Gu HP. Study on blood coagulation and hepatoprotective effect of Phlomis Maximowiczii. Regel and blood coagulation of Psoralea Coryylifolia L. Henan Sheng, China: Henan University; 2014.

[22] Chan KC, Yin MC, Chao WJ. Effect of diallyl trisulfiderich garlic oil on blood coagulation and plasma activity of anticoagulation factors in rats. Food Chem Toxicol. 2007;45:502-507.

[23] Lou CQ, Pang ZR, Zhang W, et al. Discussion of experiment study and mechanism of stanchingin Chinese Herb. J Liaoning Univ TCM. 2009;11(10):59-61.

[24] Jin GL, Chen C. Atithrombotic effects of Toona sinensis seeds decotion. China Pharm. 2011;22 (15):1364-1366.

[25] Faggio C, Sureda A, Morabito S, et al. Flavonoids and platelet aggregation: a brief review. Eur J Pharmacol. 2017;807:91-101.

[26] Trischitta F, Faggio C. Effect of the flavonol quercetin on ion transport in the isolated intestine of the eel, Anguilla anguilla. Comp Biochem Physiol C. 2006;143:17-22.

[27] Borst O, Walker B, Münzer P, et al. Skepinone-L, a novel potent and highly selective inhibitor of p38 MAP kinase, effectively impairs platelet activation and thrombus formation. Cell Physiol Biochem. 2013;31:914-924.

[28] Zhang XT, Wang K, Zhou XH, et al. Study of Xueshuan Xinmaining Tablets,s effects on expression of NO/ET-1 
on acute blood-stasis model rats. Chin J Lab Diagn. 2012;16(9):1575-1577.

[29] Yao Y, Wu WY, Liu AH, et al. Interaction of salvianolic acids and notoginsengno-sides in inhibition of ADPinduced platelet aggregation. Am J Chin Med. 2008;36 (2):313-328.

[30] Briglia M, Antonia Rossi M, Faggio C. Eryptosis: ally or enemy. Curr Med Chem. 2017;24(9):937-942.

[31] Pagano M, Faggio C. The use of erythrocyte fragility to assess xenobiotic cytotoxicity. Cell Biochem Funct. 2015;33(6):351-355.

[32] Silva-Herdade AS, Andolina G, Faggio C, et al. Erythrocyte deformability-A partner of the inflammatory response. Microvasc Res. 2016;107:34-38.

[33] Guardiola FA, Porcino C, Cerezuela R, et al. Impact of date palm fruits extracts and probiotic enriched diet on antioxidant status, innate immune response and immune-related gene expression of European seabass (Dicentrarchus labrax). Fish Shellfish Immunol. 2016;52:298-308.

[34] Carbone D, Faggio C. Importance of prebiotics in aquaculture as immunostimulants. Effects on immune system of sparus aurata and dicentrarchus labrax. Fish Shellfish Immunol. 2016;54:172-178.

[35] Faggio C, Fazio F, Marafioti S, et al. Oral administration of Gum Arabic: effects on haematological parameters and oxidative stress markers in Mugil cephalus. Iranian J Fish Sci. 2015;14(1):60-72.

[36] Aragona M, Lauriano ER, Pergolizzi S, et al. Opuntia ficusindica (L.) Miller as a source of bioactivity compounds for health and nutrition. Nat Prod Res. Forthcoming 2017. DOI:10.1080/14786419.2017.1365073 